\title{
A Functional Representation Model Facilitating Design Space Expansion
}

\author{
Wei Xu, Ke Zhao, Yatao Li, and Peitao Cheng \\ School of Electromechanical Engineering, Xidian University, Xi'an, Shaanxi 710071, China \\ Correspondence should be addressed to Wei Xu; hntb_xuwei@yahoo.com.cn
}

Received 5 June 2013; Accepted 1 August 2013

Academic Editors: O. P. Gandhi and X.-J. Liu

Copyright (C) 2013 Wei Xu et al. This is an open access article distributed under the Creative Commons Attribution License, which permits unrestricted use, distribution, and reproduction in any medium, provided the original work is properly cited.

\begin{abstract}
This paper addresses the functional representation based on the event model. In the event model, the ontology is defined based on the theory of propositional logic to describe the connotation of the event, and the variant is defined based on the theories of domain relational calculus and set theory to express the variation range of the event, which is alterable part of the event under the constraints of the ontology. Function is an important concept in conceptual design and has its connotation and extension. The functional representation is proposed based on the event model. The ontology of event is used to describe the connotation of function and to reflect the stability of function. The variant of the event is used to represent the extension and to incarnate the variety of function. The extension of function is the change range of function under the constraints of the connotation. The proposed functional representation divides the function into the immutable part and the alterable part, facilitating the expansion of design space. A functional reasoning model is also put forward based on the event model to support the function reasoning on the computers. Finally, a simple case validates the feasibility of the model.
\end{abstract}

\section{Introduction}

Product is the realization of functions. It is the core issue of product design to study and realize functions of products. The functional representation [1] is an important method to express a product and is vital in the whole design process. It is an important research content of the design theory and methodology. The functional representation functionally abstracts and describes the market demands and user requirements by computer analysis, highlights the design task, and facilitates finding out the novel design solutions. The basic concern of functional representation is how to represent the knowledge of functions [2].

The functional representation should meet the following three conditions.

(1) The functional representation should be able to completely express the requirements of product design and should not lose information in the entire design process.

(2) The functional representation should be convenient to realize the mapping and transformation functions to behaviours and structures.
(3) The functional representation should contain constraint information. Constraints play a very important role in any design. The functional representation relies on constraints to limit the search space of design.

In addition, the function serves as an important concept in the conceptual design and must have its connotation and extension. The connotation of a function describes the nature of a function and is the foundation of the function surface, the distinction between a function and another function, and also the foundation of the information exchange about this function. The extension of a function describes the variation range of the function and is the variable part of a function, which reflects the variety of function and is the content of the information communication. For the innovative conceptual design, the functional representation also needs to be able to fully reflect the connotation and extension of a function and guarantees that the function has a larger variable solution space for designers to easily associate and expand the design solutions.

The functional representation supporting the association and expansion is to ensure that the designers can find 
as much as possible functional design information related to the design problem in the design process, providing designers with greater solving space of the design problem. It will be more conducive to the development of the creative thinking of the designers and then get more innovative design solutions. The traditional functional representations only pay attention to the above three conditions, so the design results are often limited in a smaller range, and, as a result, it is difficult to get innovative design results. Therefore, the representation of the connotation and extension of function should be investigated. Not only can the functional representation fully describe design requirements, but also the connotation and extension information of function can be easily applied to the design process.

This paper discusses the representation of the connotation and extension of function and proposes the functional representation method based on the event model. The organization of the rest of the paper is as follows. Section 2 introduces the related work on the functional representation and the event and regards the event as an important knowledge representation method. Section 3 analyzes the relationship between the event and the function from the point of view of the philosophic thinking and the product constitution and regards the event as a knowledge representation method to represent the function. Section 4 defines the ontology and the variant to represent the event and establishes the event model. Section 5 proposes the functional representation based on the event model. Section 6 puts forward the functional reasoning model. The feasibility on expanding the design space of the models is verified with a simple case in Section 7. Section 8 ends the paper with some conclusions.

\section{The Literature Review}

2.1. Functional Representations. So far, there are various functional representation methods, and the typical methods include the input-output transformation model [36], the function-behaviour-structure model [7-10], and the function-behaviour-state model [11-14].

The input-output transformation model defined function as the input-output transformation relation of the physical quantities such as energy, material, and information. This type of representation has been widely used by design researchers.

The function-behaviour-structure model defines function, structure, and behaviours as three classes of properties of a design object. Function properties dictate its intended purpose and requirements; structure properties represent the description of the whole and its constituents, while the behaviour properties spell out how the structure of the object achieves its function. The description object of this model is the design instance, and it highlights the existence of the structure in the instance.

The characteristic of the function-behaviour-state model is to convert the decomposition of a function into that of behaviour description, and thus behaviour is decomposed into a series of states. The change between any two states is just the action result of a subbehaviour, while the two states often correspond to a basic physical model. The description object of this model is the abstract physical model, and it does not describe the concrete structure.

Moreover, many researchers do a lot of useful work on functional representation.

Chandrasekaran and Josephson [15] used the top-down method to express function. They first described the whole function of equipment and then described the behaviour of each component based on the function. Iwasaki et al. [16] extended this method and proposed a causal functional representation language to express equipment functions.

Sasajima et al. [17] put forward the method and ontology to express components from the point of view of function and behaviour. Their function and behaviour modelling language did not depend on the vocabulary of specific areas, in which each component could be described at different abstract levels.

Szykman et al. [18] thought that function and behaviour were two different aspects of a design object, and a function could be achieved by many physical entities, and each physical entity could have different behaviour. The function drove design, and the design object had corresponding behaviour to meet the function. They put forward an expression of function and flow and designed the ontology of function and flow. However, they did not involve the computer expression and semantic representation of function and flow.

TRIZ $[19,20]$ proposed the representation of materialfield analysis, in which all functions could be broken down into three basic components which could be expressed as two substances and a field. A graphics mode is used to express function of designed system and a variety of conflicts in the design process.

The above functional representations can be classified into the behaviour-centric methods and device-centric methods. No matter what will be the centre, these functional representations are all based on the analysis of the relationship among device, action, and behaviour, rarely from the viewpoint of the connotation and extension of functions. However, the connotation and extension are important for a concept such as function, so it is a problem worthy of study to discuss the functional representation from the viewpoint of the connotation and extension of functions.

2.2. Related Works on Event. Events represent an important part of our perceivable world. Many cognitive scientists believe that people experience and cognize the objective world based on the unit of events, in accordance with the natural cognition rules of people [21]. For example, the philosophers delegated by Whitehead [22] proposed the event theory: the world is composed of the always flowing, interrelated, and fleeting events. Recently, the concept of the event has been gradually adopted in the knowledge processing domains of computational linguistics [23], information retrieval [24], information extraction [25], automatic summarization [26], natural language understanding [27], and other fields. Indeed, numerous research efforts have been devoted to this idea in recent years. 
From the applications of the events in the above knowledge processing domains, it can be seen that all these existing event-handling methods study the verb and its attributes constituting an event. Though they all study the events in natural language, for example, the natural language function representation method [28-30], studying events is instructive for applying the event theories to the functional representation.

Though many scholars have presented the definitions of the event from the needs of their research domains, yet the event does not have a uniform definition temporarily. In the language processing domain, WordNet [31] has given the broad definition that the event was something that happens at a given place and time. HowNet [32] took the event as a kind of concept and used it to distinguish the relation among concepts. Chung and Timberlake [33] defined the event as the nomenclature that was composed of three parts: the predication, the event frame, and the event boundary. Tenny and Pustejovsky [34] put forward the definition of the event circumfusing the verb and its attributes from the point of view of semantic understanding. In information retrieval and information extraction domain, Allan et al. [35] and Yang et al. [36] regarded the event as the topic, which was subdivided to be used for retrieval. In the automatic summarization domain, Filatova and Hatzivassiloglou [37] defined the atomic event, which was composed of a verb and the main components of behavior (such as participants, location, and time) that the verb connected to.

It can be seen from the definitions of the event in various domains that the event-based studies often focused on the verbs and their attributes. These definitions not only paid more attention to the verbs but also took into account the relation between the verbs and their correlative components and extended the event to the more abstract level of the event class. The event-based studies took the event as a way of knowledge representation, which was different from the concept-based knowledge representation and was the important complement to the concept-based knowledge representation.

The basic concern of functional representation is how to represent knowledge about function. The best way is to find a convenient knowledge representation method. As for the above analysis, the event is a basic syntax and semantic unit and also is a promising way of knowledge representation. Therefore, it will be heuristic to represent function on the level of the event. This paper regards the event as a syntax structure with a core of verb-noun chunk in the language representation form and thinks of the event as the accident occurring in a special space and time in semantics. This paper attempts to study the functional representation from the viewpoint of events.

\section{Event in Product}

3.1. Philosophic Thinking of Event. Whether it has been cognized by human or not, the objective things in the world always exist objectively. Among them a part of objective things has been cognized; there are always concepts to contrast these things in the cognition. And human usually uses their connotation and extension to describe these concepts in the language. That is, things, concepts, their connotation, and extension are related to the objective world, the information world, and the language world, which are the three usually involved levels through which people cognize and describe the world.

The connections among the objective things are various, in which an important connection means that one objective thing acts on another objective thing. In this connection, not only the things and the action are important, but also the factors affecting action are indispensable. All these can form a connected whole, then can be represented with a concept of event at the information level, and can be described by the ontology and variant of the event at language level. In this description, the connection of objective things, the event, and the ontology and variant of the event are also involved in the objective world, the information world, and the language world.

As we know the products are only a part of the objective things, and the particularity of the products is that they are the man-made things. The function of the product is just the cognition of people in the information world, and the functional representation of the product is the knowledge representation in the language world. From the above analysis, we can find it is possible to use event to represent product function. The relations among event, natural language, and product function can be described with Figure 1.

In Figure 1, it can be seen from the levels of the three worlds that the process from above to below is a process of cognition, and the process from below to above is that of representation. It also can be seen from each level of the three worlds that the relationship from left to right is the relationship of combination, and the relationship from right to left is that of conceptualization. It is obvious that event plays a role of connecting link between the preceding and the following, so the event in functional representation should be researched.

3.2. Events Constitutions of Product. Human society is moving forward in the continuous innovation process of watching and using events. For instance, Faraday discovered electromagnetic induction phenomenon in 1831. Based on this phenomenon, Faraday built the first prototype generator which could produce electric power. The phenomenon of electromagnetic induction can be described with the event of electromagnetic induction. By expanding the ontology of this event and decreasing its variant, people have invented a lot of power generation equipments to generate electricity. For example, DC generators and alternators were invented to fulfill our various needs. Hydroelectric generators, wind generators, and diesel generators were invented according to generator-driven modes. Based on the reverse application of electromagnetic induction, a lot of electrical equipments have been invented to generate other forms of energy used to drive other equipments, such as motors, electric cars, 


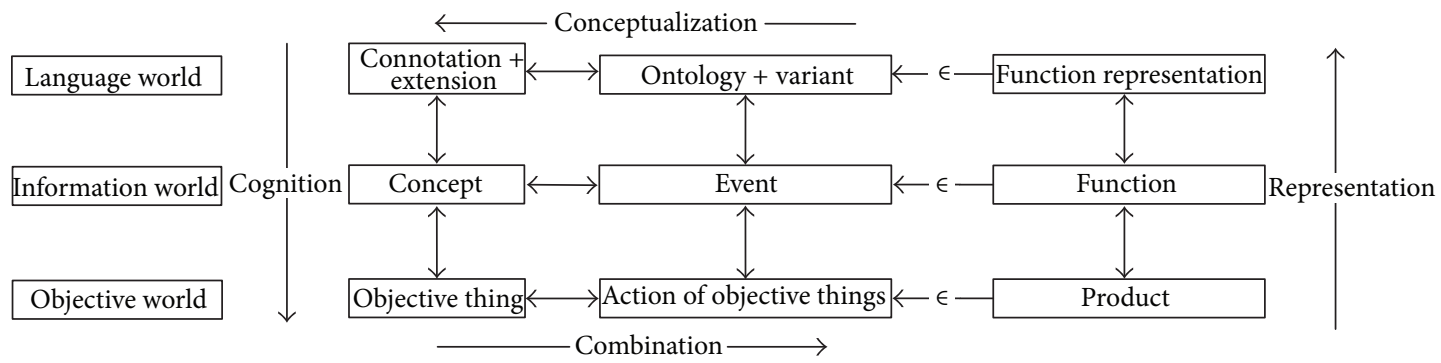

FIGURE 1: The relationship between event and function.

electronic sensor accelerators, electromagnetic guns, and magnetic levitation trains.

The view of studying problems from the perspective of event could be extended to the product design field. People have invented a lot of products to service the human life, and in essence these products are to make people's lives be more orderly and controlled. For these products to play roles, on the one hand they must dock with the events of people's life directly or indirectly, so that these life events can be automatic or semiautomatic, which makes the human life events achieved by products; on the other hand, for various products which can serve the people's life events to achieve their intended function, they must also be scheduled to make its internal contained controlled events carried through orderly, only in this way they can become mature products.

Any product with certain functions contains a series of events. A product can be considered as a system. The system is composed of subsystems which organically connect together in space and time forming a system. A subsystem is a system itself which also can be decomposed into components and actions. Components and actions are the basic units that constitute a system and also the composing elements of the events behind products. Shafts, gears, spline, boxes, and so forth are the examples of components; grasp, release, rotation, transport, and so forth are the examples of actions. Components constitute the static structure of a system, and actions constitute the dynamic behaviours of the system. Both the static structures and dynamic behaviours organically form a system to complete a preconcerted task and achieve a scheduled event, namely achieve a certain product functions to meet certain needs of people.

Therefore, product design is actually the study of the events contained behind the product, which not only include specific compositions of a product but also, through the realization of events, achieve functions of a product and facilitate the abstract description of products.

\section{Event Model}

An event consists of two parts, namely, ontology and variant. The ontology of an event describing the nature and connotation of the event is the foundation of the event surface, the distinction between an event and another one, and also the foundation of the information exchange about this event. The variant of an event describes the variation range of the event, which is an alterable part of the event under the constraints of the ontology, reflects diversity of an event, and is the content of the information communication.

The representation of ontology is based on the theory of propositional logic, while the representation of variant is based on the theory of domain relational calculus and set theory.

4.1. Ontology of Event. The ontology of an event is a set of all facts about this event. The facts can be described as propositions, so they can be represented by a series of always valid propositional formulas. These propositions not only reflect the truth of the logic but also reflect the truth of the facts. Accordingly, the proposition in the ontology of an event is called the valid proposition. The research results about the propositional logic and its reasoning all can be applied to the ontology of an event.

Definition 1 (basic valid propositions). A basic valid proposition is a basic description about an event and consists of a group of propositions. In this group, any proposition cannot be deduced from the other propositions in the group, and all other propositions not in this group can be deduced from the propositions in the group.

Suppose the basic valid propositions in the ontology $Q$ of the event $E$ are $q_{1}, q_{2}, \ldots, q_{i}, \ldots, q_{n}$; then

$$
\begin{aligned}
& Q \longrightarrow q_{1} \wedge q_{2} \wedge \cdots \wedge q_{i} \wedge \cdots \wedge q_{n} \\
& q_{1} \wedge q_{2} \wedge \cdots \wedge q_{i} \wedge \cdots \wedge q_{n} \longrightarrow Q
\end{aligned}
$$

Formula (1) shows that, when the ontology of an event is true, any proposition in the ontology is true. It is a kind of reasoning method in the daily life, and this reasoning belongs to deductive reasoning. Table 1 shows the ontology of a pulley drive event. In this table, when an event is confirmed to be a pulley drive event, any basic proposition is true.

Formula (2) indicates that the basic valid propositions in the ontology constitute the basic existing conditions of the event. The increase and decrease in quantity and the destruction of the truth of these propositions will all destroy the original event and may produce new event. For instance, when the second proposition in Table 1 does not exist, pulley drive event will not happen.

Formula (2) also provides a method of identifying events which is the foundation of the identification and diagnosis 
TABLE 1: Ontology of pulley drive event.

\begin{tabular}{ll}
\hline Number & Proposition in the ontology \\
\hline 1 & $\begin{array}{l}\text { Inherits the ontology of the friction drive event. } \\
\text { Possesses the driving pulley with the diameter } D_{1} \text { and } \\
\text { the rotational speed } n_{1} \text {, and } D_{1}>0, n_{1} \geq 0 .\end{array}$ \\
3 & $\begin{array}{l}\text { Possesses the driven pulley with the diameter } D_{2} \text { and } \\
\text { the rotational speed } n_{2} \text {, and } D_{2}>0, n_{2} \geq 0 .\end{array}$ \\
4 & Possesses $N$ belts with the length $L$, and $N \geq 1, L>0$. \\
5 & Works by the friction between the pulleys and belts. \\
6 & Drive ratio is $R$. \\
6.1 & $R=D_{1} / D_{2}$. \\
6.2 & $R=n_{1} / n_{2}$. \\
$\vdots$ & $\vdots$
\end{tabular}

method. If we confirm that all the basic valid propositions in the ontology of an event are true, we can identify the event. For example, when we ensure that all the propositions in Table 1 are true, we can assert that this event is the pulley drive event.

Definition 2 (equivalent valid proposition). An equivalent valid proposition can be deduced from the basic valid propositions and is the equivalent description of the basic valid proposition in some aspects of an event.

The equivalent valid proposition can be expressed as "can also be described as" in natural language. For instance, the valid proposition 6.1 in Table 1 can also be described as valid proposition 6.2 .

Definition 3 (ontology of event). Suppose the basic valid propositions in the ontology $Q$ of the event $E$ are $q_{1}, q_{2}$, $\ldots, q_{i}, \ldots, q_{n}$, and the corresponding equivalent valid propositions $q_{i}$ are $q_{i 1}, q_{i 2}, \ldots, q_{i m}$; the ontology $Q$ can be described as

$$
Q \longleftrightarrow q_{1} \wedge q_{2} \wedge \cdots \wedge\left(q_{i 1} \wedge q_{i 2} \wedge \cdots \wedge q_{i m}\right) \wedge \cdots \wedge q_{n}
$$

Formula (3) is the continuation of formula (1) and formula (2). On the one hand, it shows that in the reasoning from the ontology of an event to the propositions not only the basic valid propositions but also the correlative equivalent valid proposition can be deduced. On the other hand, the related event can also be identified by the equivalent valid propositions.

The proposition in the ontology of an event is ever true and does not change, but the concepts that constitute the proposition are not unchangeable. For example, in Table 1, the belts in proposition 4 have many forms. As long as a form can meet the requirements of the belt in proposition 4 , a designer can choose which kind of form to use, which will not affect the truth of the proposition in the pulley drive event. So the requirements of the truth of the ontology on this event level can be satisfied, and also enough feasible space to the events or concepts in next level can be provided. In product design, designers can use the projection operation of event, under the condition of guaranteeing the truth of ontology, to constantly improve the product.
TABLE 2: Variant of pulley drive event.

\begin{tabular}{ll}
\hline Number & Attributes of variant \\
\hline 1 & $\begin{array}{l}\text { Specific values of the parameters of the driving pulley, } \\
\text { such as the values of } D_{1} \text { and } n_{1} .\end{array}$ \\
2 & $\begin{array}{l}\text { Specific values of the parameters of the driven pulley, } \\
\text { such as the values of } D_{2} \text { and } n_{2} .\end{array}$ \\
3 & The length $L$ and the number $N$ of the belts. \\
4 & $\begin{array}{l}\text { The distance } D \text { between the driving pulley and driven } \\
\text { pulley. }\end{array}$ \\
$\vdots$ & $\vdots$
\end{tabular}

4.2. Variant of Event. The variant of an event not only describes the change range of a variable in the event but also overall expresses the change range of the event. Based on the theory of domain relational calculus and set theory, the definition of the variant of the event $E$ is given as following.

Definition 4 (variant of event). Suppose $V$ is the variant of event $E$. The attributes of variant $V$ are composed of $v_{1}, v_{2}, \ldots, v_{k}$, the corresponding attribute variables are $d_{1}, d_{2}, \ldots, d_{k}$, and the related value sets are $D_{1}, D_{2}, \ldots, D_{k}$. A Cartesian product space is formed with these value sets: $D=D_{1} \times D_{2} \times \cdots \times D_{k}$. Let $D^{\prime}$ be a subset satisfying the constraints of the ontology $Q, D^{\prime} \subseteq D$, where $D^{\prime}$ is the change range of event $E$, which is also called the variant. Let $d^{k}=\left(d_{1}, d_{2}, \ldots, d_{k} \mid\left(E\left(v_{1}: d_{1}, v_{2}: d_{2}, \ldots, v_{k}: d_{k}\right)\right) \wedge\left(d_{1} \in\right.\right.$ $\left.\left.D_{1} \wedge d_{2} \in D_{2} \wedge \cdots \wedge d_{k} \in D_{k}\right)\right)$ be a point of event $E$, and the change range of $d^{k}$ reflects the change range of variant.

The variables of a variant can be the continuous variables or discrete variables and also can be a mixture of both. When the variable range contains infinite number of values, the Cartesian product space is an infinite set, whose subset can be infinite sets, such as the variant of the pulley drive event (see Table 2), or can be a finite set.

4.3. Relationship between Ontology and Variant. The ontology and variant of an event, respectively, describe the connotation and extension of the event. The ontology not only is a full description of the connotation but also is the integrated constraints to the variant. The constraints can be the value constraint for a single variable; for example, in Table 1 the diameter $D_{1}$ of the pulley should meet $D_{1}>0$ and may be the constraint involved in the description of several variables, for example, the calculation formula of the drive ratio in Table 1.

Due to the linear correlation of the constraints of the ontology, the Cartesian product space that is composed of the variables of the variant may cause degradation phenomenon. For example, there are four variables to describe the size of the pulley drive device, namely the diameter $D_{1}$ of driving pulley, the diameter $D_{2}$ of driven pulley, the distance $D$ between the pulley and the length $L$ of the belt, in which only three variables are independent, namely, the diameter $D_{1}$, the diameter $D_{2}$, and one of the distance $D$, and the length $L$ can be determined with the size of the pulley drive device. So the four-dimensional Cartesian product space will degrade to the 
three-dimensional space. From this perspective, a variant is the feasible space of an event under the constraints of the ontology.

The ontology of an event describes the inner nature of the event. For a specific product, it must have abundant connotation. But it is not better for a product to have abundant connotation, because the abundant connotation of a product is at the cost of the reduction of its extension. The more abundant connotation a product has, the stronger the constraint to the extension will become. Synchronously, the lower the adaptability of the product to the external environment is, the narrower the range of application will be. Figure 2 takes the mechanical drive events as an example to demonstrate the relationship between the ontology and variant of the event.

4.4. Event Model. According to the above analysis, the ontology of an event not only gives the complete description of the connotation of the event but also establishes the relationship between the event and its subevents. The unified logic description is used to make the operation of the ontology relatively simple and to make it have the mature theory basis. The application of the set theory and domain relational calculus is convenient for us to describe the variant of event, and the establishment of Cartesian product space also makes us have a complete understanding of the extension of an event.

Combining with the definition of ontology and variant, the complete definition of an event is given as following.

Definition 5 (event). Suppose $Q$ is the ontology of event $E$, and $V$ is the variant of event $E$. Let $q_{1}, q_{2}, \ldots, q_{i}, \ldots, q_{n}$ be the formulas for the basic valid propositions of the event, and let $q_{i 1}, q_{i 2}, \ldots, q_{i m}$ be the formulas for the equivalent valid propositions of $q_{i}$. Then, the ontology can be represented as follows: $Q \leftrightarrow q_{1} \wedge q_{2} \wedge \cdots \wedge\left(q_{i 1} \wedge q_{i 2} \wedge \cdots \wedge q_{i m}\right) \wedge \cdots \wedge q_{n}$. The attributes of variant $V$ are composed of $v_{1}, v_{2}, \ldots, v_{k}$, the corresponding attribute variables are $d_{1}, d_{2}, \ldots, d_{k}$, and the related value sets are $D_{1}, D_{2}, \ldots, D_{k}$. A Cartesian product space is formed with these value sets: $D=D_{1} \times D_{2} \times \cdots \times$ $D_{k}$. Letting $D^{\prime}$ be a subset that satisfies the constraints of basic valid propositions $Q, D^{\prime} \subseteq D$. Here, $D^{\prime}$ is the change range of event $E$, which is also called the variant. Let $d^{k}=$ $\left(d_{1}, d_{2}, \ldots, d_{k} \mid\left(E\left(v_{1}: d_{1}, v_{2}: d_{2}, \ldots, v_{k}: d_{k}\right)\right) \wedge\left(d_{1} \in\right.\right.$ $\left.\left.D_{1} \wedge d_{2} \in D_{2} \wedge \cdots \wedge d_{k} \in D_{k}\right)\right)$ be a point of event $E$. Then, $\left(Q,\left(d_{1}, d_{2}, \ldots, d_{k} \mid\left(E\left(v_{1}: d_{1}, v_{2}: d_{2}, \ldots, v_{k}: d_{k}\right)\right) \wedge\left(d_{1} \in\right.\right.\right.$ $\left.\left.\left.D_{1} \wedge d_{2} \in D_{2} \wedge \ldots \wedge d_{k} \in D_{k}\right)\right)\right)$ is a specific event of event $E$. The latter is represented as $E=\left\{Q,\left[d^{k} \mid d^{k} \in D^{\prime} \subseteq D\right]\right\}$.

The ontology and variant of event describe the event from different angles. In each angle only a part of an event is described, and the combination of the two parts forms a full description of the event. The variant is actually the range of an event under the constraints of the ontology of the event.

In product design, the application value of events not only lies in the truth and stability of its ontology but also depends on the adaptation range of its variant to the external environment. The truth and stability of the ontology are
TABLE 3: Common attributes of the event.

\begin{tabular}{ll}
\hline Attribute name & Function \\
\hline Agent & Describing the agent and its value set \\
Object & Describing the object and its value set \\
Participant & Describing the participant and its value set \\
Action & Describing the operation and its value set \\
Time & Describing the time and its value set \\
Place & Describing the place and its value set \\
Reason & Describing the reason and its value set \\
Purpose & Describing the purpose and its value set \\
Means & Describing the means and its value set \\
Result & Describing the result and its value set \\
Condition & Describing the condition and its value set \\
Process & Describing the process and its value set \\
\hline
\end{tabular}

the foundation of cognizing and applying the event. The value of the ontology depends on all the propositions in it. Because all the propositions in the ontology correspond and cooperate, an event can exploit its whole advantage and comprehensive advantage, overcome the limitation of a sole proposition, and make the application value of the event much higher than that of its any internal proposition.

The usage of events can be divided into two ways: one is using the ontology of events, and the other is synchronously using the ontology and variant of events. The first way is to use one or a set of propositions of the ontology. The second way is to use some propositions of the ontology to meet partial needs of a design problem and then to determine the variables of the variant in order to solve the further needs of the problem, which is the common way for a product design.

\section{Functional Representation Model}

5.1. Functional Representation. The function is an important concept that represents product, which is constituted by connotation and extension. As analyzed in Section 3.2, the event can be adopted to represent the function. By defining the ontology of an event to describe the connotation of function and defining the variant of an event to describe the extension of a function, we can establish the functional representation model based on the event model. This functional representation model divides the functional representation into the unchangeable connotation and alterable extension, in which the alterable part actually is the change range under the constraints of the unchangeable part. Thus the design space can be expediently expanded, making the design results more innovative.

5.1.1. Representation of Extension. The extension of a function is represented with the variant of an event. Based on the event model, the variant of an event is composed of the event attributes, and each event attribute has a corresponding attribute variable. These event attribute variables have their respective value sets and reflect the range of the variant by the Cartesian product space formed by these value sets. Therefore, determining the attributes of a variant is the key 


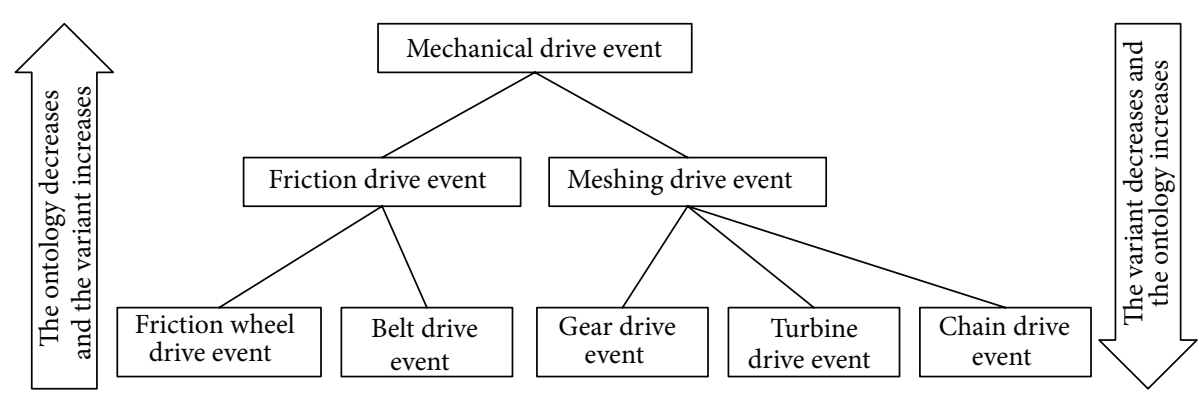

FIGURE 2: Example of relationship between ontology and variant.

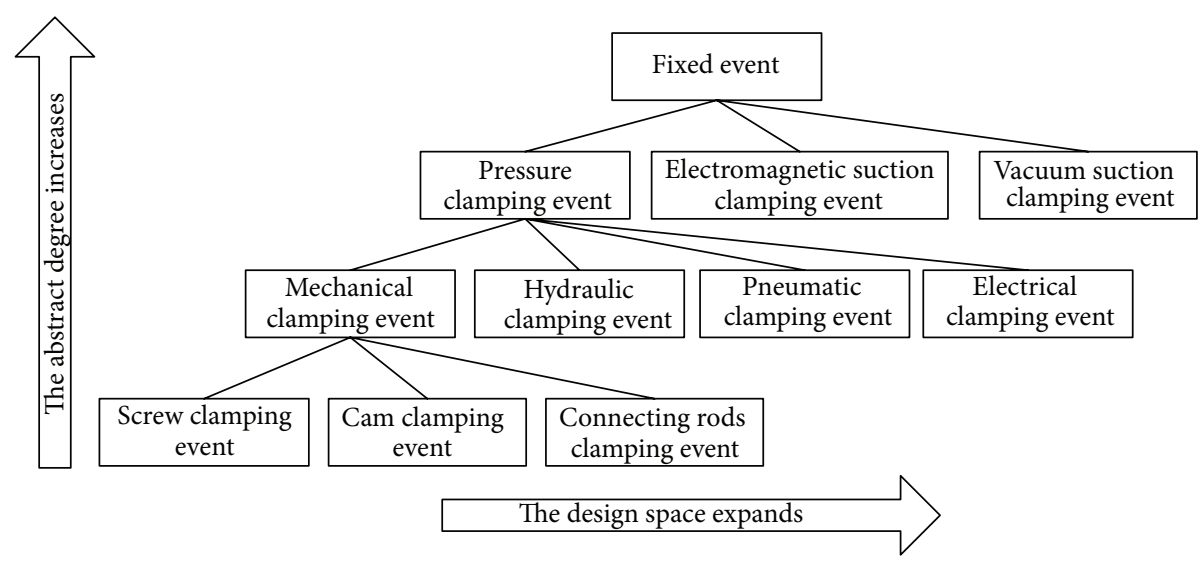

FIGURE 3: Abstract degree of the design plans.

problem of adopting the variant of an event to represent the extension of a function.

A product can be as simple as having only one action and one component and also can be as complex as including thousands of actions and components. However, a basic function of a product is usually achieved by the components performing an action. There are many factors influencing the action, and the common factors include the technical areas, the types of components, and the space and time in which the action occurs. Actually, all these components, actions, and influencing factors need to be considered in the product design.

When representing the product function with the event, we can take into account the components, actions, and various factors together, regard them as the event attributes, and integrate them into the variant of an event to form a specific event. The event attributes highlight the relation among components, actions, and influencing factors and can express the composition and function of a product. The more comprehensive understanding by which people described the relevant factors of the event, the more reasonable the products will be designed.

The event attributes are divided into the common attributes and private attributes. The common attributes are the attributes that all the events possess. These attributes will exist when the events occur, and sometime some of them are not designated just because of the inheritance or default. The common attributes of the event are shown in Table 3. The private attributes are the special attributes that special events possess, and different events have the different private attributes. For example, the event describing object motion would have the private attribute of speed.

5.1.2. Representation of Connotation. The connotation of a function is represented with the ontology of an event. Based on the event model, the ontology of an event is the set of all the facts about this event. The facts can be described as propositions, so they can be represented by a series of ever valid propositional formulas. After determining the attributers of the variant of an event, the valid propositions of the ontology of an event can be described with the propositional formulas that are composed of the event attributers, logical operators, arithmetic operators, and comparison operators. The expressive effect of the connotation of a function depends on the designers' acquisition and cognition of the design requirements and design resources and plays an important role in the consequent design.

5.2. Representation of Functional Knowledge. The basic concern of functional representation is how to represent the knowledge of a function. Based on the event model, the functional knowledge is divided into the abstract event knowledge and the instance event knowledge. They are the important knowledge in the product design.

5.2.1. Abstract Event Knowledge. The abstract event knowledge is the commonsense knowledge which describes a kind 
of events, which is the abstraction result of this kind of events, and has a certain common recognition. The abstract event knowledge has the same internal structure and the common characteristics abstracted from a large number of real events existing in the objective world. It is mainly summarized from the aspect whether the event attributes, such as agents, objects, and actions satisfy the ontology constraints of the concrete events. The result of the summarization can be thought of as an abstract event.

Definition 6 (abstract event). Suppose $Q$ is the ontology of event $E$, and $V$ is the variant of event $E$. The attributes of variant $V$ are composed of the attributes such as agent, object, participant, action, time, place, means, reason, purpose, result, condition, process, and private attribute. Then the event possessing the constraint $F$ is referred to as abstract event and can be represented as follows:

$$
\begin{gathered}
E=\left\{Q,\left(d_{0}, d_{1}, \ldots, d_{12} \mid\right.\right. \\
E\left(\text { action: } d_{0}, \text { agent: } d_{1}, \text { object: } d_{2},\right. \\
\text { participant: } d_{3} \text {, time: } d_{4}, \\
\text { place: } d_{5}, \text { means: } d_{6} \text {, reason: } d_{7}, \\
\text { purpose: } d_{8}, \text { result: } d_{9}, \text { condition: } d_{10}, \\
\text { process: } \left.\left.d_{11}, \text { private attribute: } d_{12}\right)\right) \\
\left.\wedge F\left(d_{0}, d_{1}, \ldots, d_{12}\right)=\text { TRUE }\right\} \\
\left\{Q \wedge F\left(d_{0}, d_{1}, \ldots, d_{12}\right)=\right.\text { TRUE, } \\
\left(d_{0}, d_{1}, \ldots, d_{12} \mid\right. \\
E\left(\text { action: } d_{0}, \text { agent: } d_{1},\right. \\
\text { object: } d_{2}, \text { participant: } d_{3}, \text { time: } d_{4}, \\
\text { place: } d_{5}, \text { means: } d_{6}, \text { reason: } d_{7}, \\
\text { purpose: } d_{8}, \text { result: } d_{9}, \text { condition: } d_{10}, \\
\text { process: } \left.\left.\left.d_{11}, \text { private attribute: } d_{12}\right)\right)\right\} .
\end{gathered}
$$

In this definition, in order to expediently describe the event knowledge, in this paper "private attribute" only stands for one of the private attributes of an event. Certainly, an event may have many private attributes, and more corresponding private attributes need be appended according to the practical problem. This assumption is used in the rest of the paper.

Abstract event describes the constraints to various attributes of the event. A set of values of these attributes which satisfy the constraints to various attributes of the event is an instantiation to the abstract event, and it can create the corresponding instance event.

Examples are as follows:

(1) Chuck clamps steel tube.

(2) Graduator clamps hilt.

(3) Vise clamps bolt.
The three design examples all describe the kind of events that tongs clamp workpieces. The event can be described in the form of abstract event as follows:

$$
\begin{gathered}
E=\left\{Q \wedge \left(d_{0} \in \text { tongs } \wedge d_{1} \in \text { workpiece } \wedge d_{2}=\text { clamp } \wedge d_{8}\right.\right. \\
=(\text { The location of the workpiece is fixed by } \\
\text { tongs } \wedge \text { the movement patterns of } \\
\text { workpiece is consistent with the tongs })), \\
\left(d_{0}, d_{1}, \ldots, d_{12} \mid\right. \\
E\left(\text { action: } d_{0} \text {, agent: } d_{1}, \text { object: } d_{2},\right. \\
\text { participant: } d_{3}, \text { time: } d_{4}, \\
\text { place: } d_{5}, \text { means: } d_{6}, \text { reason: } d_{7}, \\
\text { purpose: } d_{8}, \text { result: } d_{9}, \text { condition: } d_{10}, \\
\text { process: } \left.\left.\left.d_{11} \text {, privateattribute: } d_{12}\right)\right)\right\} .
\end{gathered}
$$

5.2.2. Instance Event Knowledge. Instance event knowledge is the instantiated knowledge describing a specific event, which is the result of instantiation produced by a specific event in accordance with the constraints of abstract event knowledge. An instance event is generated from an abstract event. As long as a specific action, components, and the affected factors satisfy the constraints of the ontology of an abstract event, an instance event can be generated to express the actual situation according to the abstract event.

The above abstract event that tongs clamp workpieces can be used to solve the practical workpiece fixation problem that rotary table clamps semifinished product, which can be described in the form of the instance event as follows:

$E=\left\{Q \wedge\left(d_{0}=\right.\right.$ rotary table $\wedge d_{1}=$ semifinished product

$$
\begin{aligned}
& \wedge d_{2}=\text { clamp } \wedge d_{8} \\
& =(\text { The location of the semifinished product }
\end{aligned}
$$

is fixed by rotary table

$\wedge$ the movement patterns

of semifinished product is consistent

with the rotary table)),

$$
\begin{gathered}
\left(d_{0}, d_{1}, \ldots, d_{12} \mid\right. \\
E\left(\text { action: } d_{0} \text {, agent: } d_{1} \text {, object: } d_{2},\right. \\
\text { participant: } d_{3} \text {, time: } d_{4}, \\
\text { place: } d_{5}, \text { means: } d_{6},
\end{gathered}
$$




$$
\begin{aligned}
& \text { reason: } d_{7} \text {, purpose: } d_{8} \text {, } \\
& \text { result: } d_{9} \text {, condition: } d_{10} \text {, } \\
& \text { process: } \left.\left.\left.d_{11} \text {, private attribute: } d_{12}\right)\right)\right\} \text {. }
\end{aligned}
$$

\subsubsection{Relation between Abstract Event and Instance Event.} The relationship between the abstract event and instance event is an abstract-specific relationship. The abstract event is the abstract event knowledge frame that is abstracted from plenty of instance events with the same internal structure and the common characteristics. It is the knowledge of the instance event that knowledge aims at the colony of events and is provided with the abstraction, generality, and recognition. The instance event is the description of a specific event that occurred at special time, place, and status. It represents a specific instance of an event, focusing on an individual of an event.

The process of abstracting the abstract event knowledge from large numbers of instance events is the induction process. By finding the resemblance and difference of the instance events and carrying through the classification and abstraction, we can obtain the abstract event knowledge that describes these instance events.

The process of generating an instance event from the abstract event knowledge is the deductive process. The abstract event knowledge provides the frame and the range for the instance event. Under the constraints of the ontology of an abstract event, assigning each attribute of the event variant with a specific value can produce an instance event that meets the needs.

\section{Reasoning Model of Event}

The event model uses the ontology and variant to solve the knowledge representation of an event. However, it is important to make the event knowledge play the corresponding important roles in product development process. This involves how to handle the event knowledge and how to combine the event knowledge with practical design problems. This section discusses the reasoning model of an event.

6.1. Selection of Event. A product design team or designer must have a lot of event knowledge of the product design. Suppose the knowledge is enough to guide a certain design problem, and there is also a lot of event knowledge that is not related to the design problem. How to select the event knowledge that is useful for the design problem and discard the temporarily useless knowledge? This is actually a selection problem of event knowledge.

Selecting the event knowledge related to a practical design problem depends on the practical problem, which is achieved by summing up the known information of the practical problem and expressing it in the form of one or more propositions. These propositions are usually the basic valid propositions and the propositional formulas whose operation objects are constants or the variables of the variant. These propositions are the foundation of selection operation.

In the selection operation of event knowledge, the extraction operation between the above propositions and the ontology of all the event knowledge should be executed. This expands the ontology of event, then through the constraint function of the ontology acts on the variant to narrow the variant, and ultimately gets the needed event knowledge.

Definition 7 (selection of event). Suppose $Q$ is the ontology of event $E$, and $V$ is the variant of event $E$. The attributes of variant $V$ are composed of $v_{1}, v_{2}, \ldots, v_{k}$, and the corresponding attribute variables are $d_{1}, d_{2}, \ldots, d_{k}$. Suppose $F$ is a set of propositional formulas whose operation objects are constants or the variables of the variant, and the operators are logic operators or comparative operators. Then the selection operation (denoted by $\sigma_{F}(E)$ ) of the event $E$ about $F$ is defined as follows:

$$
\begin{gathered}
\sigma_{F}(E)=\left\{Q,\left(d_{1}, d_{2}, \ldots, d_{k} \mid\right.\right. \\
\left.E\left(v_{1}: d_{1}, v_{2}: d_{2}, \ldots, v_{k}: d_{k}\right)\right) \\
\wedge\left(Q,\left(d_{1}, d_{2}, \ldots, d_{k} \mid\right.\right. \\
\left.\left.E\left(v_{1}: d_{1}, v_{2}: d_{2}, \ldots, v_{k}: d_{k}\right)\right)\right) \\
\left.\in E \wedge F\left(d_{1}, d_{2}, \ldots, d_{k}\right)=\mathrm{TRUE}\right\} \\
=\left\{Q \wedge F\left(d_{1}, d_{2}, \ldots, d_{k}\right)=\mathrm{TRUE},\right. \\
\left(d_{1}, d_{2}, \ldots, d_{k} \mid E\left(v_{1}: d_{1}, v_{2}: d_{2}, \ldots, v_{k}: d_{k}\right)\right) \\
\wedge\left(Q \wedge F\left(d_{1}, d_{2}, \ldots, d_{k}\right)=\mathrm{TRUE},\right. \\
\left.\left.\left(d_{1}, d_{2}, \ldots, d_{k} \mid E\left(v_{1}: d_{1}, v_{2}: d_{2}, \ldots, v_{k}: d_{k}\right)\right) \in E\right)\right\},
\end{gathered}
$$

where $F\left(d_{1}, d_{2}, \ldots, d_{k}\right)$ is a proposition formula about the variables $d_{1}, d_{2}, \ldots, d_{k}$ of the variant.

From the above definition of selection, we can see that the results of selection is an event set, but this set is a subset of the event set $E$ before selection. It can be described as follows: if $E^{\prime}=\sigma_{F}(E)$, then $E^{\prime} \subseteq E$. It is obvious that using the selection of event we can limit the event knowledge needed, so as to determine the processing scope of the event knowledge and to improve the processing efficiency of the event knowledge.

6.2. Projection of Event. The result of the selection of event is an event set, so that we can obtain the event knowledge where each individual event is a unit and can understand the whole of these events. If we wish to further understand and operate these events, we must execute the projection operation of event.

In the event model the ontology and variant are adopted to represent an event. The ontology and variant of an event provide the foundation to reveal the internal structure of event, from which we can comprehend the local and details of an event. The projection of event is divided into 
the proposition projection of the ontology and the attribute projection of the variant.

The proposition projection of the ontology is the operation to select one or more valid propositions from the designated ontology. The result of the proposition projection is the set that is composed of the corresponding valid propositions. The proposition projection of event ontology is defined as follows.

Definition 8 (proposition projection). Suppose $Q$ is the ontology of event $E$, and $V$ is the variant of event $E$. Let $q_{1}, q_{2}, \ldots, q_{i}, \ldots, q_{n}$ be the formulas for the basic valid propositions of the event. The attributes of variant $V$ are composed of $v_{1}, v_{2}, \ldots, v_{k}$, and the corresponding attribute variables are $d_{1}, d_{2}, \ldots, d_{k}$. Then the proposition projection (denoted by $\left.\pi_{A}(E(Q))\right)$ of the ontology $Q$ about the set $A$ composed of the specified number of propositions is expressed as follows:

$$
\begin{aligned}
& \pi_{A}(E(Q)) \\
& =\left\{q_{A} \mid Q \longleftrightarrow q_{1} \wedge q_{2} \wedge \ldots \wedge q_{n},\right. \\
& \quad\left(d_{1}, d_{2}, \ldots, d_{k} \mid E\left(v_{1}: d_{1}, v_{2}: d_{2}, \ldots, v_{k}: d_{k}\right)\right) \\
& \wedge\left(Q,\left(d_{1}, d_{2}, \ldots, d_{k} \mid\right.\right. \\
& \left.\left.\left.E\left(v_{1}: d_{1}, v_{2}: d_{2}, \ldots, v_{k}: d_{k}\right)\right)\right) \in E\right\},
\end{aligned}
$$

where $A$ is the set that is composed of the specified number of the valid propositions of the event ontology, which is the subset of the corpora $\{1,2, \ldots, n\}$ composed of all the number of the valid propositions of the event ontology.

The attribute projection of the variant is the operation to select one or more attribute variables from the designated variant. The result of the attribute projection is the set composed of the corresponding attribute variables. The attribute projection of the variant is defined as follows.

Definition 9 (attribute projection). Suppose $Q$ is the ontology of event $E$, and $V$ is the variant of event $E$. The attributes of variant $V$ are composed of $v_{1}, v_{2}, \ldots, v_{k}$, and the corresponding attribute variables are $d_{1}, d_{2}, \ldots, d_{k}$. Then the attribute projection (denoted by $\pi_{A}(E(V))$ ) of the variant $V$ about the set $A$ composed of the specified number of attributes is expressed as follows:

$$
\begin{aligned}
& \pi_{A}(E(V))=\left\{d_{A} \mid Q,\right.\left(d_{1}, d_{2}, \ldots, d_{k} \mid\right. \\
&\left.E\left(v_{1}: d_{1}, v_{2}: d_{2}, \ldots, v_{k}: d_{k}\right)\right) \\
& \wedge\left(Q,\left(d_{1}, d_{2}, \ldots, d_{k} \mid\right.\right. \\
&\left.\left.\left.E\left(v_{1}: d_{1}, v_{2}: d_{2}, \ldots, v_{k}: d_{k}\right)\right)\right) \in E\right\},
\end{aligned}
$$

where $A$ is the set that is composed of the specified number of the attributes of the event variant, which is the subset of the corpora $\{1,2, \ldots, k\}$ composed of all the number of the attributes of the event variant.
The above two definitions show that we can recognize and operate the event from the two parts of the ontology and variant of an event, and the prerequisites are to ensure the validation of the original ontology.

6.3. Instantiation of Event. In product design, when the event knowledge needed and enough cognition on the details of the event knowledge are acquired, we can start to apply the event knowledge to the product design. This process is to transform the relatively abstract event knowledge into the specific event that can be executed by the product. This process is a combination of theory and practice and is also a process from abstract to specific.

The operation of transforming the abstract event into the specific event is the instantiation of event. Because the process that acquires the abstract event is performed under the constraint of the ontology, the instantiation of event only requires to instantiate the variables of the variant. The attribute instantiation of an event is defined as follows.

Definition 10 (attribute instantiation). Suppose $Q$ is the ontology of event $E$, and $V$ is the variant of event $E$. The attributes of variant $V$ are composed of $v_{1}, v_{2}, \ldots, v_{k}$, the corresponding attribute variables are $d_{1}, d_{2}, \ldots, d_{k}$, the related value sets are $D_{1}, D_{2}, \ldots, D_{k}$, and any value selection of $d_{i}$ from $D_{i}$ is written as $\lambda\left(d_{i}\right)$. Then the attribute instantiation (denoted by $\left.\lambda_{i}(E)\right)$ of the event $E$ is expressed as follows:

$$
\begin{aligned}
\lambda_{i}(E)= & \left\{Q,\left(d_{1}, d_{2}, \ldots, d_{i}, \ldots, d_{k} \mid\right.\right. \\
& \left.E\left(v_{1}: d_{1}, v_{2}: d_{2}, \ldots, v_{i}: d_{i}, \ldots, v_{k}: d_{k}\right)\right) \\
\wedge & \left(Q,\left(d_{1}, d_{2}, \ldots, d_{i}, \ldots, d_{k} \mid\right.\right. \\
& \left.\left.E\left(v_{1}: d_{1}, v_{2}: d_{2}, \ldots, v_{i}: d_{i}, \ldots, v_{k}: d_{k}\right)\right)\right) \\
\in & \left.E \wedge d_{i}=\lambda\left(d_{i}\right)\right\} \\
= & \left\{\wedge d_{i}=\lambda\left(d_{i}\right),\right. \\
& \left(d_{1}, d_{2}, \ldots, d_{i}, \ldots, d_{k} \mid\right. \\
\left.E\left(v_{1}: d_{1}, v_{2}: d_{2}, \ldots, v_{i}: d_{i}, \ldots, v_{k}: d_{k}\right)\right) & \wedge\left(Q \wedge d_{i}=\lambda\left(d_{i}\right),\right. \\
& \left(d_{1}, d_{2}, \ldots, d_{i}, \ldots, d_{k} \mid\right. \\
& \left.\left.\left.E\left(v_{1}: d_{1}, v_{2}: d_{2}, \ldots, v_{i}: d_{i}, \ldots, v_{k}: d_{k}\right)\right)\right) \in E\right\} .
\end{aligned}
$$

If each variable of the event variant is instantiated, the event will become a specific event. This event operation is referred to as the instantiation of event. The instantiation of event is defined as follows.

Definition 11 (instantiation). Suppose $Q$ is the ontology of event $E$, and $V$ is the variant of event $E$. The attributes of variant $V$ are composed of $v_{1}, v_{2}, \ldots, v_{k}$, the corresponding attribute variables are $d_{1}, d_{2}, \ldots, d_{k}$, the related value sets are $D_{1}, D_{2}, \ldots, D_{k}$, and each value selection of $d_{i}$ from $D_{i}$ is 
written as $\lambda\left(v_{i}\right)$. Then the instantiation (denoted by $\lambda(E)$ ) of the event $E$ is expressed as follows:

$$
\begin{aligned}
& \lambda(E)=\{Q,\left(d_{1}, d_{2}, \ldots, d_{i}, \ldots, d_{k} \mid\right. \\
& E\left(v_{1}: d_{1}, v_{2}: d_{2}, \ldots,\right. \\
&\left.\left.v_{i}: d_{i}, \ldots, v_{k}: d_{k}\right)\right) \\
& \wedge\left(Q,\left(d_{1}, d_{2}, \ldots, d_{i}, \ldots, d_{k} \mid\right.\right. \\
&\left.\left.E\left(v_{1}: d_{1}, v_{2}: d_{2}, \ldots, v_{i}: d_{i}, \ldots, v_{k}: d_{k}\right)\right)\right) \\
& \in E \wedge d_{1}=\lambda\left(d_{i}\right) \wedge d_{2}=\lambda\left(d_{2}\right) \\
&\left.\wedge \cdots \wedge d_{i}=\lambda\left(d_{i}\right) \wedge \ldots \wedge d_{k}=\lambda\left(d_{k}\right)\right\} \\
&\left\{Q \wedge d_{1}=\lambda\left(d_{1}\right) \wedge d_{2}=\lambda\left(d_{2}\right) \wedge \ldots \wedge d_{i}=\lambda\left(d_{i}\right)\right. \\
& \wedge \cdots \wedge d_{k}=\lambda\left(d_{k}\right), \\
&\left(d_{1}, d_{2}, \ldots, d_{i}, \ldots, d_{k} \mid\right. \\
&\left.E\left(v_{1}: d_{1}, v_{2}: d_{2}, \ldots, v_{i}: d_{i}, \ldots, v_{k}: d_{k}\right)\right) \\
& \wedge\left(Q \wedge d_{1}=\lambda\left(d_{1}\right) \wedge d_{2}=\lambda\left(d_{2}\right)\right. \\
& \wedge \cdots \wedge d_{i}=\lambda\left(d_{i}\right) \wedge \ldots \wedge d_{k}=\lambda\left(d_{k}\right), \\
&\left(d_{1}, d_{2}, \ldots, d_{i}, \ldots, d_{k} \mid\right. \\
&\left.\left.\left.E\left(v_{1}: d_{1}, v_{2}: d_{2}, \ldots, v_{i}: d_{i}, \ldots, v_{k}: d_{k}\right)\right)\right) \in E\right\} .
\end{aligned}
$$

Obviously, with the event instantiated operation, we can transform the abstract event knowledge into more specific event, so as to gradually eliminate the uncertainty contained in the abstract event knowledge, making the event more realizable.

6.4. Connection of Events. The selection, projection and instantiation of event are the operations of the individual event, which are mainly the refining operations of event and in which the relationship processing among the events is not involved. In fact, a product is usually composed of numerous components which interact and contact with each other in order to realize the scheduled functions of the product. The interaction contact of the components can be viewed as events. These events are independent from the angle of individuals namely, they can be distinguished from each other. However, these events are interactional and harmonious from the overall view. Therefore, the product can be regarded as the whole of the events that connected the components. These events play an important role for the product to achieve its scheduled functions. Accordingly, it is necessary to discuss the relation operation between the events.

The connection of events is one of the important relation operations of event, which is the description of the interaction between the two events under certain conditions. The connection of events is defined as follows.
Definition 12 (connection of events). Suppose event $E$ and event $R$ are mutually independent, and $Q_{E}$ and $Q_{R}$ are, respectively, the ontology of events $E$ and $R$. Let $\left(d_{E 1}, d_{E 2}, \ldots, d_{E k} \mid\right.$ $\left.E\left(v_{E 1}: d_{E 1}, v_{E 2}: d_{E 2}, \ldots, v_{E k}: d_{E k}\right)\right)$ be a point of event $E$ $\left(d_{R 1}, d_{R 2}, \ldots, d_{R h} \mid E\left(v_{R 1}: d_{R 1}, v_{R 2}: d_{R 2}, \ldots, v_{R h}: d_{R h}\right)\right)$ a point of event $R$. Then the connection of event $E$ and $R$ is expressed as follows:

$$
\begin{aligned}
& E \times R \\
& =\left\{Q_{E} \wedge Q_{R},\right. \\
& \quad\left(d_{1}, d_{2}, \ldots, d_{m} \mid\right. \\
& \quad\left(d_{E 1}, d_{E 2}, \ldots, d_{E k} \mid\right. \\
& \left.\quad E\left(v_{E 1}: d_{E 1}, v_{E 2}: d_{E 2}, \ldots, v_{E k}: d_{E k}\right)\right) \\
& \quad \times\left(d_{R 1}, d_{R 2}, \ldots, d_{R h} \mid\right. \\
& \left.\quad R\left(v_{R 1}: d_{R 1}, v_{R 2}: d_{R 2}, \ldots, v_{R h}: d_{R h}\right)\right) \\
& \wedge\left(Q_{E},\left(d_{E 1}, d_{E 2}, \ldots, d_{E k} \mid\right.\right. \\
& \left.\left.\quad E\left(v_{E 1}: d_{E 1}, v_{E 2}: d_{E 2}, \ldots, v_{E k}: d_{E k}\right)\right)\right) \in E \\
& \wedge\left(Q_{R},\left(d_{R 1}, d_{R 2}, \ldots, d_{R h} \mid\right.\right. \\
& \left.\left.\left.\left.R\left(v_{R 1}: d_{R 1}, v_{R 2}: d_{R 2}, \ldots, v_{R h}: d_{R h}\right)\right)\right) \in R\right)\right\} .
\end{aligned}
$$

In the definition, the independence of the event means that the negative propositions of the valid propositions in $Q_{E}$ and $Q_{R}$ cannot be deduced from $Q_{E} \wedge Q_{R}$ nor can the new propositions of $Q_{E} \wedge Q_{R}$ and the propositions that are different from $Q_{E}$ and $Q_{R}$.

Definition 13 (attribute connection of events). Suppose event $E$ and event $R$ are mutually independent, and $Q_{E}$ and $Q_{R}$ are, respectively, the ontology of events $E$ and $R$. Let $\left(d_{E 1}, d_{E 2}, \ldots, d_{E k} \mid E\left(v_{E 1}: d_{E 1}, v_{E 2}: d_{E 2}, \ldots, v_{E k},: d_{E k}\right)\right) \mathrm{a}$ point of event $E$ and $\left(d_{R 1}, d_{R 2}, \ldots, d_{R h} \mid E\left(v_{R 1}: d_{R 1}, v_{R 2}\right.\right.$ : $\left.\left.d_{R 2}, \ldots, v_{R h}: d_{R h}\right)\right)$ a point of event R. $\theta$ is the arithmetic comparison operator. Then the attribute connection of the $i$ th attribute of the variant of event $E$ and the $j$ th attribute of the variant of event $R$ about $\theta$ operation is expressed as follows:

$$
\begin{gathered}
E \theta R=\left\{Q_{E} \wedge Q_{R} \wedge d_{E i} \theta d_{R j},\right. \\
\left(d_{1}, d_{2}, \ldots, d_{m} \mid\right. \\
\left(d_{E 1}, d_{E 2}, \ldots, d_{E i}, \ldots, d_{E k} \mid\right. \\
E\left(v_{E 1}: d_{E 1}, v_{E 2}: d_{E 2}, \ldots,\right. \\
\left.\left.v_{E i}: d_{E i}, \ldots, v_{E k}: d_{E k}\right)\right)
\end{gathered}
$$




$$
\begin{gathered}
\times\left(d_{R 1}, d_{R 2}, \ldots, d_{R j}, \ldots, d_{R h} \mid\right. \\
R\left(v_{R 1}: d_{R 1}, v_{R 2}: d_{R 2}, \ldots,\right. \\
\left.\left.v_{R j}: d_{R j}, \ldots, v_{R h}: d_{R h}\right)\right) \\
\wedge\left(d_{E 1}, d_{E 2}, \ldots, d_{E i} \ldots, d_{E k} \mid\right. \\
E\left(v_{E 1}: d_{E 1}, v_{E 2}: d_{E 2}, \ldots,\right. \\
\left.\left.v_{E i}: d_{E i}, \ldots, v_{E k}: d_{E k}\right)\right) \\
\in\left(d_{R 1}, d_{R 2}, \ldots, d_{R j} \ldots, d_{R h} \mid\right. \\
R\left(v_{R 1}: d_{R 1}, v_{R 2}: d_{R 2}, \ldots,\right. \\
\left.\left.\left.\left.v_{R j}: d_{R j}, \ldots, v_{R h}: d_{R h}\right)\right) \in R\right)\right\},
\end{gathered}
$$

where $d_{E i} \theta d_{R j}$ represents the proposition of the $i$ th attribute of the variant of event $E$ and the $j$ th attribute of the variant of event $R$ about $\theta$ operation which is true.

From the above definitions, it can be seen in the connection operation of events that the ontology of the events executes the conjunction operation, which increases the facts of the ontology of the connected event. The variants of the event execute the Cartesian product operation, which expands the feasible space of the variant. Therefore, the implementation of the connection of events is not only the process of increasing the new propositions about the nature and functions in the ontology but also the process of expanding the change range of the variant.

The connection of events also presents a kind of method to describe the relationship between events at a relative abstract level. Although the connected events are the relatively independent events, at a relative abstract level, we often use a relatively abstract event to describe connection results and discuss the events at different levels according to their demands. For example, the driving pulley drive belt event is composed of two events: the driving pulley rotate event and the driving pulley frictionize belt event. The variant of driving pulley rotate event has the attribute of the driving pulley, and the driving pulley frictionize belt event also has the attribute of the driving pulley. Through the same attribute, the attribute connection operation of the two events can be executed, and the result of the connection is just the driving pulley drive belt event. Therefore, sometime this relative abstract event can be used to represent the two specific events.

A product usually contains many events. It is just through the connection of events that can make the product work according to the scheduled functions and achieve the value of the product.

6.5. Decomposition of Event. The design process of a product can be divided into two categories: one is the integration process from bottom to top, from details to whole; the other is the decomposition process from top to bottom, from whole to details. Of course the two kinds of methods are not entirely separated, and most of the time they are used alternately.

If the design task, subtask, and details can be seen as the different events, the integration process of design is the process of connecting events, and the decomposition process of design is the process of decomposing events.

The decomposition of an event is the inverse process of connecting the events. An event can be resumed by connecting the subevents that are decomposed from the original event. The decomposition of an event is defined as follows.

Definition 14 (decomposition of event). Suppose $Q$ is the ontology of event $E$, and $Q$ can be decomposed as $Q \leftrightarrow$ $Q_{R} \wedge Q_{S} \wedge d_{R i} \theta d_{S j}$, where $Q_{R}$ and $Q_{S}$ are two independent sets of propositions and $d_{R i} \theta d_{S j}$ is the proposition that describes the relationship between the common attribute variables of the decomposed sub-events. $V$ is the variant of event $E$. The attributes of variant $V$ are composed of $v_{1}, v_{2}, \ldots, v_{k}$, and the related value sets are $D_{1}, D_{2}, \ldots, D_{k}$. The attribute variables can be classified into two kinds: the attribute variables set $\left\{d_{R 1}, d_{R 2}, \ldots, d_{R m}\right\}$ under the constraints of $Q_{R}$ and the attribute variables set $\left\{d_{S 1}, d_{S 2}, \ldots, d_{S h}\right\}$ under the constraints of $Q_{S}$, and these sets meet the condition: $\left\{d_{R 1}, d_{R 2}, \ldots, d_{R m}\right\} \cap$ $\left\{d_{S 1}, d_{S 2}, \ldots, d_{S h}\right\} \neq \Phi$. Then event $E$ can be decomposed into the independent sub-events $R$ and $S$. The decomposition of event $E$ is expressed as follows:

$$
\begin{aligned}
& E=\left\{Q,\left(d_{1}, d_{2}, \ldots, d_{k} \mid R\left(v_{1}: d_{1}, v_{2}: d_{2}, \ldots, v_{k}: d_{k}\right)\right)\right\} \\
& =\left\{Q \longleftrightarrow Q_{R} \wedge Q_{S} \wedge d_{R i} \theta d_{S j},\right. \\
& \left(d_{R 1}, d_{R 2}, \ldots, d_{R i}, \ldots, d_{R m}, d_{S 1}\right. \text {, } \\
& d_{S 2}, \ldots, d_{S j}, \ldots, d_{S h} \mid \\
& R\left(v_{R 1}: d_{R 1}, v_{R 2}: d_{R 2}, \ldots,\right. \\
& v_{R i}: d_{R i}, \ldots, v_{R m}: d_{R m}, v_{S 1}: d_{S 1} \text {, } \\
& \left.\left.v_{S 2}: d_{S 2}, \ldots, v_{S j}: d_{S j}, \ldots, v_{S h}: d_{S h}\right)\right) \\
& \wedge\left\{v_{R 1}, v_{R 2}, \ldots, v_{R i}, \ldots, v_{R m}\right\} \\
& \cup\left\{v_{S 1}, v_{S 2}, \ldots, v_{S j}, \ldots, v_{S h}\right\} \\
& =\left\{v_{1}, v_{2}, \ldots, v_{k}\right\} \\
& \wedge\left\{v_{R 1}, v_{R 2}, \ldots, v_{R i}, \ldots, v_{R m}\right\} \\
& \left.\cap\left\{v_{S 1}, v_{S 2}, \ldots, v_{S j}, \ldots, v_{S h}\right\} \neq \Phi\right\} \\
& =\left\{Q_{R},\left(d_{R 1}, d_{R 2}, \ldots, d_{R h} \mid\right.\right. \\
& \left.\left.R\left(v_{R 1}: d_{R 1}, v_{R 2}: d_{R 2}, \ldots, v_{R h}: d_{R h}\right)\right)\right\} \\
& \times\left\{Q_{S},\left(d_{S 1}, d_{S 2}, \ldots, d_{S h} \mid\right.\right. \\
& \left.\left.S\left(v_{S 1}: d_{S 1}, v_{S 2}: d_{S 2}, \ldots, v_{S h}: d_{S h}\right)\right)\right\}=R \times S .
\end{aligned}
$$




\section{A Simple Case Study}

The realization of any design objective is all achieved through the completion of a certain function therefore, the design objective can be abstractly defined as functions. We can adopt the product conceptual design with the main body of functional representation and implementation instead of the product entity and take this as the starting point and basis to seek the innovative design scheme. This would be beneficial to expand the design ideas and avoid the limitations of the product entity design.

A procedure for functional representation to expand the design space based on event model is expressed as follows.

(1) Analyse the design objective and find out an event to describe the objective.

(2) Based on the design objective and design knowledge, construct the ontology and the variant of the event to give the functional representation of the design objective.

(3) Change the attributes of the variant of the event under the constraints of the ontology of the event to expand the design space.

(4) Obtain the valuable solutions of the design objective in the above expansion process.

For example, a design objective is to design a kind of device to clamp a workpiece in with a machine tool.

This design objective can be represented with the functional representation based on the event model, and can be described with a clamping event. The ontology of the event can be acquired from the design objective and design knowledge. From the design knowledge, it is known that the ontology of the clamping event should inherit the ontology of the fixed event. From the design requirement, it is known that its agent attribute is the device to design, its object attribute is the workpiece, its action attribute is "clamp", its place attribute is the machine tool, and its aim attribute is to fix the workpiece. The variant of the clamping event is composed of the common attributes and the private attributes which come from the finally design plan. Based on the functional representation of this paper, the clamping event $E$ can be expressed as follows:

$$
\begin{gathered}
E=\left\{Q \wedge \left(d_{0} \in \text { device to design } \wedge d_{1}=\right.\right.\text { workpiece } \\
\wedge d_{2}=\text { clamp } \wedge d_{4}=\text { machine tool } \\
\left.\wedge d_{8}=\text { fix workpiece }\right), \\
\left(d_{0}, d_{1}, \ldots, d_{12} \mid\right. \\
E\left(\text { action: } d_{0} \text {, agent: } d_{1} \text {, object: } d_{2},\right. \\
\text { participant: } d_{3}, \text { time: } d_{4} \text {, place: } d_{5}, \\
\text { means: } d_{6} \text {, reason: } d_{7} \text {, purpose: } d_{8},
\end{gathered}
$$

$$
\text { result: } d_{9} \text {, condition: } d_{10} \text {, process: } d_{11} \text {, }
$$

$$
\text { private attribute: } \left.\left.\left.d_{12}\right)\right)\right\} \text {. }
$$

Based on the clamping event, the solutions of the design objective can be found from the following views.

(1) If the agent attribute is limited to a spiral device, the clamping event $E$ is instantiated to the screw clamping event, leading to the design plan of the screw and nut.

(2) If the agent attribute is expanded to a mechanical device, the clamping event $E$ is instantiated to the mechanical clamping event, obtaining the design plan of the cam clamping and connecting rods clamping.

(3) If the agent attribute is expanded to a pressure device, the clamping event $E$ is instantiated to the pressure clamping event, obtaining the design plan of the hydraulic clamping, pneumatic clamping, and electrical clamping.

(4) If the action attribute is expanded to the action "fix", the clamping event $E$ is instantiated to the fixed event, achieving the design plan of the electromagnetic suction and vacuum suction.

From the forming process of these design plans, it can be seen that their abstract degree is different. The relationship among these plans is shown in Figure 3.

From this design problem, we can get the following facts. When the functional representation is used to describe the design objective, there is a close relationship between the solution scope of the design objective and the abstract degree of functional representation. The higher the abstract degree of functional representation is, the greater the solution scope of the design objective will be and the more innovative design schemes of a product will be obtained. The functional representation based on the event model divides the function into the immutable connotation and alterable extension, and the extension is the alterable part of the function under the constraints of the connotation. This provides the possibility of representing function in the different abstract degree, facilitating expanding the design space.

\section{Conclusions}

Based on the analysis and application of the functional representation model and reasoning model in this paper, the following conclusions may be drawn.

(1) A new perspective of the event is provided to discuss products. On the one hand, events represent an important part of our perceivable world, the manmade products are only a small part of this world, and any product with certain functions contains a series of events. So it is practicable to use events to represent product. On the other hand, the event is a basic syntax and semantic unit and is also a promising way of knowledge representation. The concept of the event has been gradually adopted in the knowledge 
processing domains, which will be helpful to the use of the event in design. So it is reasonable to use events to represent function.

(2) A new functional representation method is proposed based on the event model. Function is an important concept in product design, and it has its connotation and extension. This paper establishes the event model, defines the ontology of event to describe the connotation of function, defines the variant of event to describe the extension of function, and proposes the functional representation method. The extension of a function is the alterable part of the function under the constraints of the connotation and will be propitious for expanding the design space.

(3) The functional reasoning model is put forward based on the event model with mature mathematical foundation, which makes the implementation of the functional representation on computers be possible.

\section{Acknowledgments}

The authors would like to acknowledge the financial support of the National Natural Sciences Foundation of China under Grant no. 51105290 and the Fundamental Research Funds for the Central Universities of China (no. K5051204015).

\section{References}

[1] B. Chandrasekaran, "Representing function: relating functional representation and functional modeling research streams," Artificial Intelligence for Engineering Design, Analysis and Manufacturing, vol. 19, no. 2, pp. 65-74, 2005.

[2] M. S. Erden, H. Komoto, T. J. Van Beek, V. D’Amelio, E. Echavarria, and T. Tomiyama, "A review of function modeling: approaches and applications," Artificial Intelligence for Engineering Design, Analysis and Manufacturing, vol. 22, no. 2, pp. 147$169,2008$.

[3] W. Rodenacker, Methodisches Konstruieren, Springer, Berlin, Germany, 1971.

[4] G. Pahl and W. Beitz, Engineering Design: A Systematic Approach, Springer, Berlin, Germany, 1998.

[5] J. Hirtz, R. B. Stone, D. A. McAdams, S. Szykman, and K. L. Wood, "A functional basis for engineering design: reconciling and evolving previous efforts," Research in Engineering Design, vol. 13, no. 2, pp. 65-82, 2002.

[6] Y. Kitamura and R. Mizoguchi, "Ontology-based description of functional design knowledge and its use in a functional way server," Expert Systems with Applications, vol. 24, no. 2, pp. 153166,2003

[7] J. S. Gero, "Design prototypes: a knowledge representation schema for design," AI Magazine, vol. 11, no. 4, pp. 26-36, 1990.

[8] L. Qian and J. S. Gero, "Function-behavior-structure paths and their role in analogy-based design," Artificial Intelligence for Engineering Design, Analysis and Manufacturing, vol. 10, no. 4, pp. 289-312, 1996.

[9] J. S. Gero and V. Kazakov, "Adaptive enlargement of state spaces in evolutionary designing," Artificial Intelligence for Engineering Design, Analysis and Manufacturing, vol. 14, no. 1, pp. 31-38, 2000 .
[10] J. S. Gero and U. Kannengiesser, "The situated functionbehaviour-structure framework," Design Studies, vol. 25, no. 4, pp. 373-391, 2004.

[11] Y. Umeda, M. Ishii, M. Yoshioka, Y. Shimomura, and T. Tomiyama, "Supporting conceptual design based on the function-behavior-state modeler," Artificial Intelligence for Engineering Design, Analysis and Manufacturing, vol. 10, no. 4, pp. 275-288, 1996.

[12] Y.-M. Deng, "Function and behavior representation in conceptual mechanical design," Artificial Intelligence for Engineering Design, Analysis and Manufacturing, vol. 16, no. 5, pp. 343-362, 2002.

[13] Y.-M. Deng, S. B. Tor, and G. A. Britton, "Abstracting and exploring functional design information for conceptual mechanical product design," Engineering with Computers, vol. 16, no. 1, pp. 36-52, 2000.

[14] Y. Shimomura, M. Yoshioka, H. Takeda, Y. Umeda, and T. Tomiyama, "Representation of design object based on the functional evolution process model," Journal of Mechanical Design, vol. 120, no. 2, pp. 221-229, 1998.

[15] B. Chandrasekaran and J. R. Josephson, "Function in device representation," Engineering with Computers, vol. 16, no. 3-4, pp. 162-177, 2000.

[16] Y. Iwasaki, M. Vescovi, and R. Fikes, "Causal functional representation language with behavior-based semantics," Applied Artificial Intelligence, vol. 9, no. 1, pp. 5-31, 1995.

[17] M. Sasajima, Y. Kitamura, M. Ikeda, and R. Mizoguchi, "A representation language for behavior and function: FBRL," Expert Systems with Applications, vol. 10, no. 3-4, pp. 471-479, 1996.

[18] S. Szykman, J. W. Racz, and R. D. Sriram, "The representation of function in computer-based design," in Proceedings of the 1999 SAME Design Engineering Technical Conference, Las Vegas, Nev, USA, September 1999.

[19] G. Altschuller, Creativity as an Exact Science: The Theory of the Solution of Inventive Problem Solving, Gordon \& Breach Science, New York, NY, USA, 1984.

[20] G. Altshuller, The Innovation Algorithm: TRIZ, Systematic Innovation and Technical Creativity, Technical Innovation Center, Worcester, Mass, USA, 1999.

[21] W. Zhou, Z. T. Liu, and Q. P. Kong, "A survey of event-based knowledge processing," Chinese Journal of Computer Science, vol. 33, no. 2, pp. 160-162, 2008.

[22] A. N. Whitehead, Process and Reality, Free Press, New York, NY, USA, 1978.

[23] A. Jansen and P. Niyogi, "Point process models for spotting keywords in continuous speech," IEEE Transactions on Audio, Speech and Language Processing, vol. 17, no. 8, pp. 1457-1470, 2009.

[24] D. Poshyvanyk, Y. Guéhéneuc, A. Marcus, G. Antoniol, and V. Rajlich, "Feature location using probabilistic ranking of methods based on execution scenarios and information retrieval," IEEE Transactions on Software Engineering, vol. 33, no. 6, pp. 420-432, 2007.

[25] S. Kara, Ö. Alan, O. Sabuncu, S. Akpnar, N. K. Cicekli, and F. N. Alpaslan, "An ontology-based retrieval system using semantic indexing," Information Systems, vol. 37, no. 4, pp. 294-305, 2012.

[26] L. Bai, S. Lao, A. F. Smeaton, N. E. O'Connor, D. Sadlier, and D. Sinclair, "Semantic analysis of field sports video using a petrinet of audio-visual concepts," Computer Journal, vol. 52, no. 7, pp. 808-823, 2009. 
[27] X. Wang, G. Hripcsak, M. Markatou, and C. Friedman, "Active computerized pharmacovigilance using natural language processing, statistics, and electronic health records: a feasibility study," Journal of the American Medical Informatics Association, vol. 16, no. 3, pp. 328-337, 2009.

[28] J. A. Buur, Theoretical approach to mechatronics design [Ph.D. thesis], Institute for Engineering Design, Technical University of Denmark, Lyngby, Denmark, 1990.

[29] C. T. Hansen, "An approach to simultaneous synthesis and optimization of composite mechanical systems," Journal of Engineering Design, vol. 6, no. 3, pp. 249-266, 1995.

[30] C. F. Kirschman, G. M. Fadel, and C. Jara-Almonte, "Classifying functions for mechanical design," Journal of Mechanical Design, vol. 120, no. 3, pp. 475-482, 1998.

[31] G. A. Miller, R. Beckwith, C. Fellbaum, D. Gross, and K. J. Miller, "Introduction to wordnet: an on-line lexical database," International Journal of Lexicography, vol. 3, no. 4, pp. 235-312, 1990.

[32] Z. D. Dong and Q. Dong, "Construction of a knowledge system and its impact on Chinese research," Chinese Journal of Contemporary Linguistics, vol. 3, no. 1, pp. 33-44, 2001.

[33] S. Chung and A. Timberlake, "Tense, aspect, and mood," Language Typology and Syntactic Description, vol. 3, pp. 202258, 1985.

[34] C. Tenny and J. Pustejovsky, "Events and the semantics of opposition," in Proceedings of Events as Grammatical Object, CSLI Publications, Stanford, Calif, USA, 2000.

[35] J. Allan, R. Papka, and V. Lavrenko, "On-line new event detection and tracking," in Proceedings of the 21st Annual International ACM SIGIR Conference on Research and Development in Information Retrieval, New York, NY, USA, 1998.

[36] Y. Yang, J. G. Carbonell, and R. D. Brown, "Learning approaches for detecting and tracking news events," IEEE Intelligent Systems, vol. 14, no. 4, pp. 32-43, 1999.

[37] E. Filatova and V. Hatzivassiloglou, "Event-based extractive summarization," in Proceedings of the ACL-2004 Workshop on Summarization, Barcelona, Spain, July 2004. 

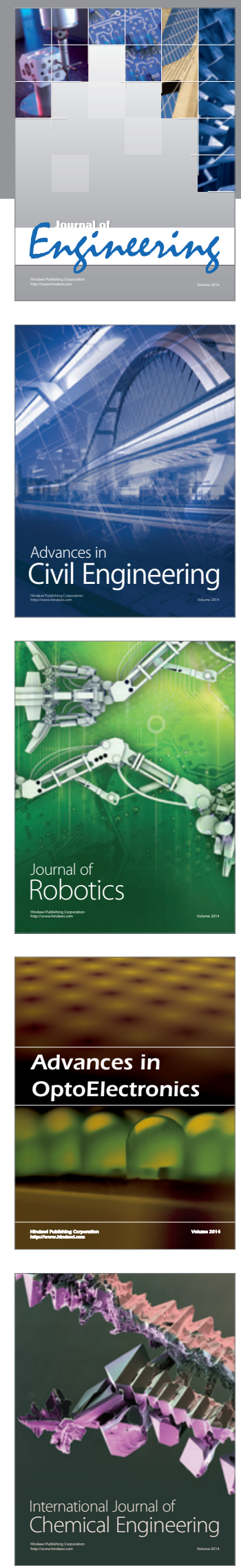

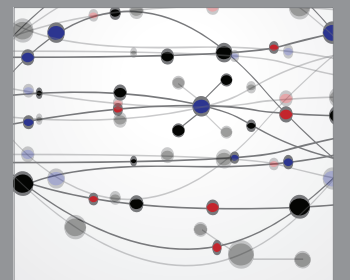

The Scientific World Journal
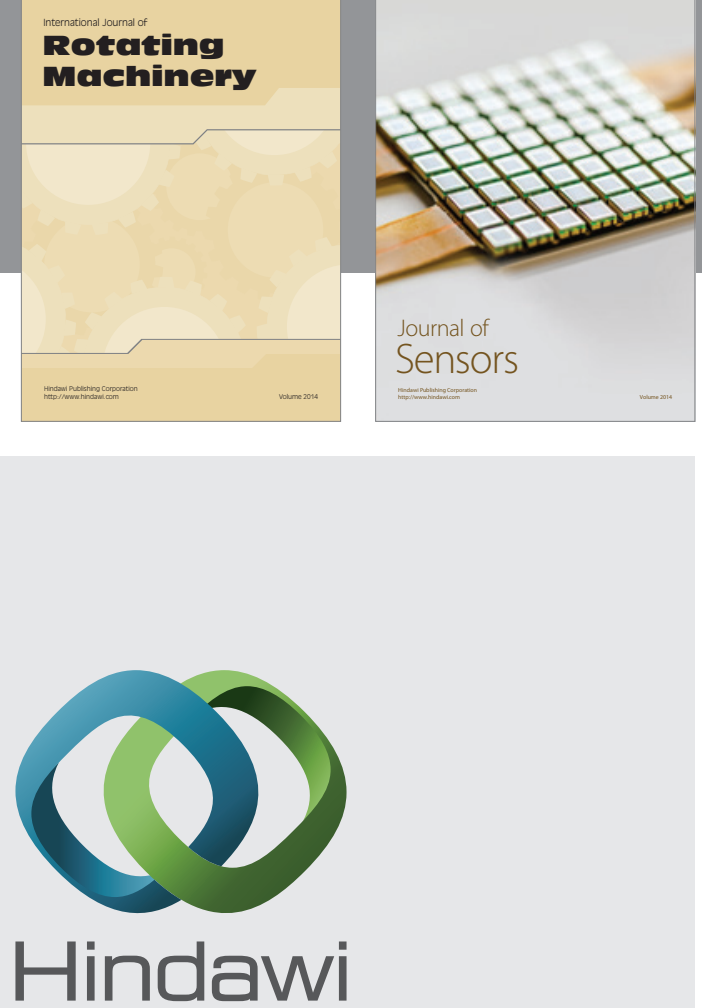

Submit your manuscripts at http://www.hindawi.com
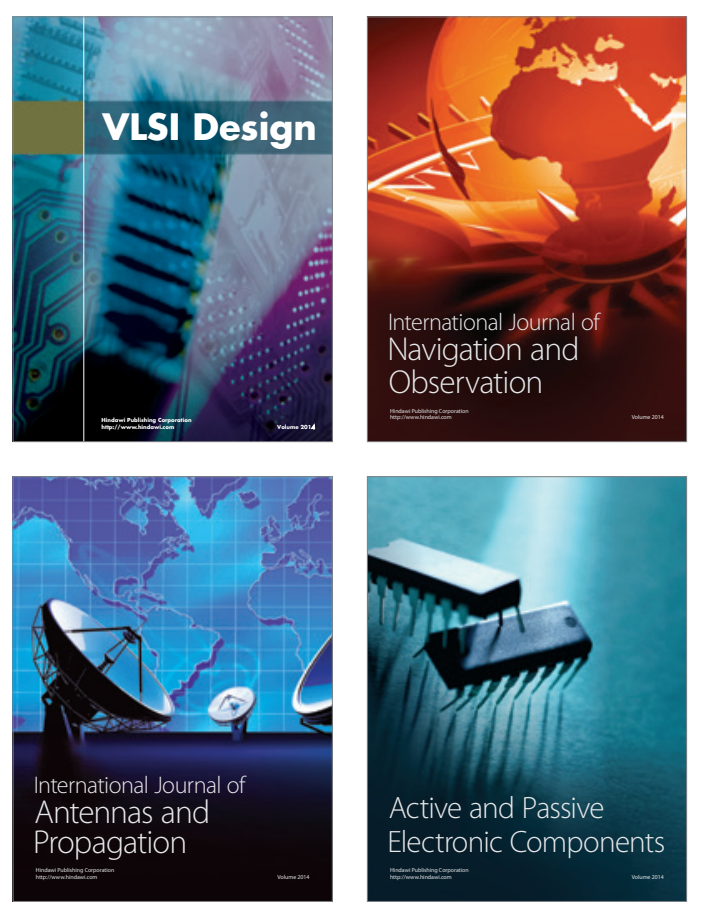
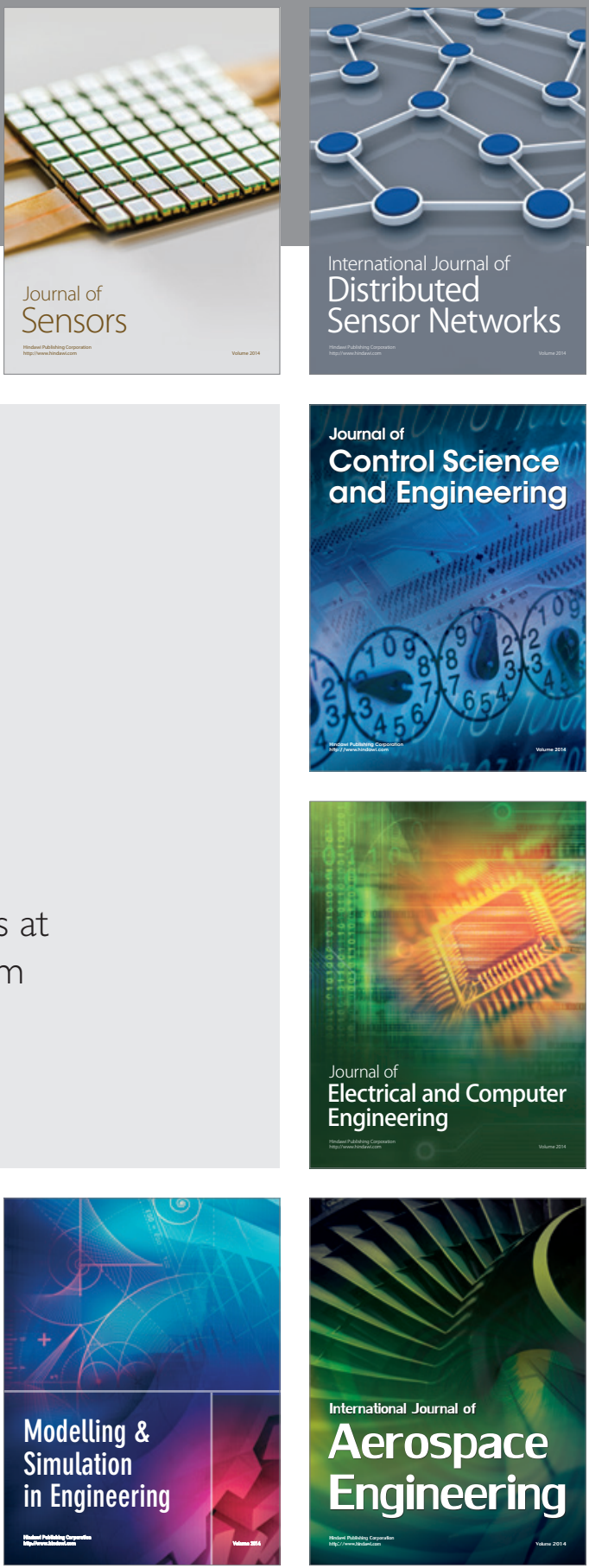

Journal of

Control Science

and Engineering
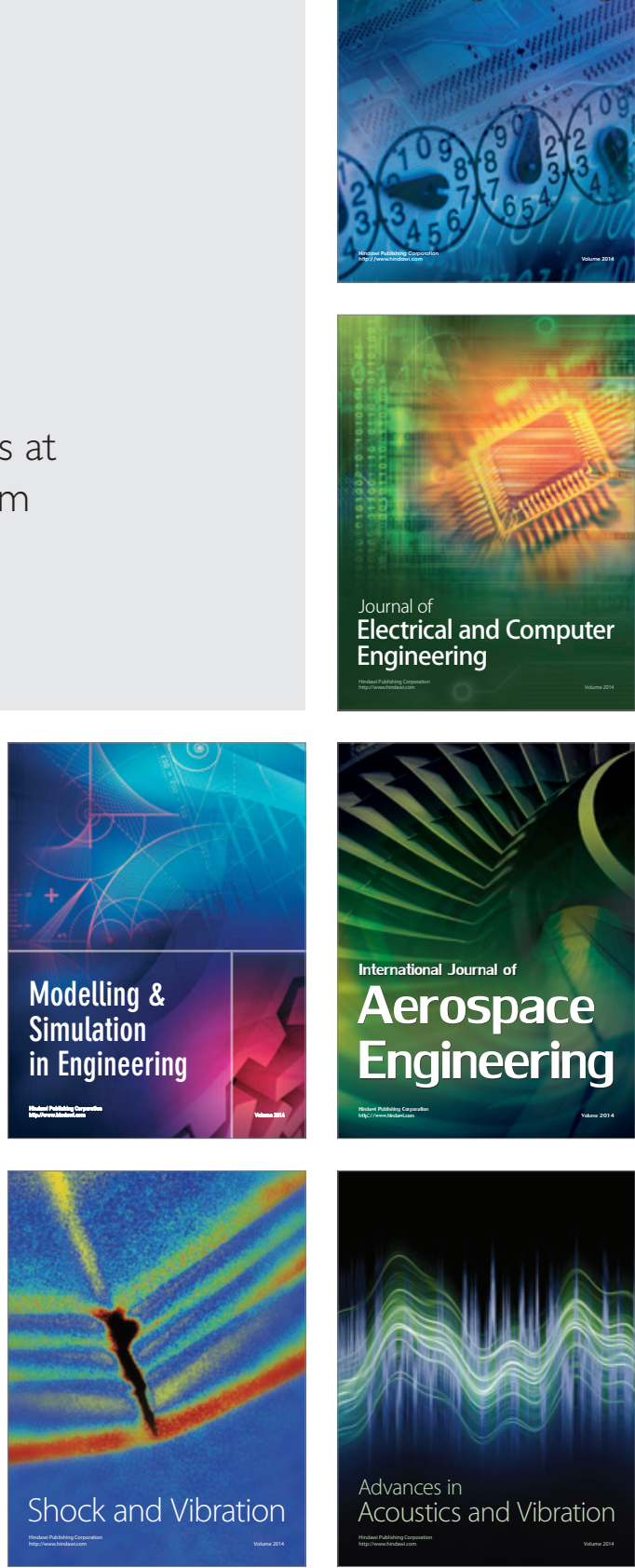